\title{
Children's Use of Geometry for Reorientation
}

\section{Citation}

Lee, Sang Ah, and Elizabeth S. Spelke. 2008. Children's use of geometry for reorientation.

Developmental Science 11(5): 743-749.

\section{Published Version}

doi:10.1111/j.1467-7687.2008.00724.x

\section{Permanent link}

http://nrs.harvard.edu/urn-3:HUL.InstRepos:10269044

\section{Terms of Use}

This article was downloaded from Harvard University's DASH repository, and is made available under the terms and conditions applicable to Open Access Policy Articles, as set forth at http:// nrs.harvard.edu/urn-3:HUL.InstRepos:dash.current.terms-of-use\#OAP

\section{Share Your Story}

The Harvard community has made this article openly available.

Please share how this access benefits you. Submit a story.

Accessibility 


\section{Children's Use of Geometry for Reorientation}

Sang Ah Lee \& Elizabeth S. Spelke

Harvard University

Correspondence: Sang Ah Lee

Department of Psychology

Harvard University

33 Kirkland Street, $11^{\text {th }}$ Floor

Cambridge, MA 02138

Phone: (609) 933-6396

E-mail: lee@wjh.harvard.edu 


\begin{abstract}
$\underline{\text { Abstract }}$
Research on navigation has shown that humans and laboratory animals recover their sense of orientation primarily by detecting geometric properties of large-scale surface layouts (e.g., room shape), but the reasons for the primacy of layout geometry have not been clarified. In four experiments, we tested whether 4-year-old children reorient by the geometry of extended wall-like surfaces because such surfaces are large and perceived as stable, because they serve as barriers to vision or to locomotion, or because they form a single, connected geometric figure. Disoriented children successfully reoriented by the shape of an arena formed by surfaces that were short enough to see and step over. In contrast, children failed to reorient by the shape of an arena defined by large and stable columns or by connected lines on the floor. We conclude that preschool children's reorientation is not guided by the functional relevance of the immediate environmental properties, but rather by a specific sensitivity to the geometric properties of the extended three-dimensional surface layout.
\end{abstract}


Human adults, children, and nonhuman animals reorient themselves primarily in accord with the geometry of the surrounding surface layout (for review, see Cheng \& Newcombe, 2005). For example, when laboratory rats are shown the location of food buried in one of the corners of a rectangular enclosure and then are disoriented, they search mostly in the two geometrically appropriate corners: the correct corner and the rotationally symmetrical opposite corner (Cheng, 1986). Human adults, young children, monkeys, birds, and fish use the shape of their environment in reorientation tasks as well (Hermer \& Spelke, 1994, 1996; Learmonth et al., 2001; Gouteux et al., 2001; Kelly et al., 1998; Vallortigara et al., 1990; Sovrano, et al., 2003). The use of non-geometric information such as wall brightness or landmark objects varies across species and tasks, but room-shape geometry is used consistently.

Nevertheless, not all environmental features with distinctive and informative geometric properties serve as a basis for reorientation. For example, rats and children reliably reorient themselves in accord with the shape of the three-dimensional enclosure in which they are tested, but often fail to reorient in accord with the shapes of two-dimensional patterns in the corners or on the walls of that enclosure (Cheng, 1986; Lourenco \& Huttenlocher, 2007). Moreover, preschool children reorient by the shape of an array marked by detached walls but not by detached corners or objects (Gouteux \& Spelke, 2001). Studies with younger toddlers further suggest that whereas unique landmarks are used to recall a location, geometric relationships between identical landmarks are not (Lew, Foster \& Bremner, 2006). These findings suggest that children and animals reorient specifically by the geometric arrangement of the extended surfaces in the environment. No study, however, yet indicates why navigating children and animals encode the geometry of some environmental features but not others.

The present experiments test three explanations for the precedence of extended surfaces in children's reorientation, each of which appeals to functional properties of the environments in which children and laboratory animals live. First, children and animals may reorient by the geometric properties of walls, but not of configurations of objects, because 
walls are perceived as stable and unmovable whereas smaller objects are not (Newcombe \& Ratliff, 2007). Landmark stability and size influence the way a variety of animals use landmarks as beacons (Biegler \& Morris, 1996; Knierim et al., 1995; Learmonth, Newcombe \& Huttenlocher, 2001), including insects, who use large columns to navigate to a known food source (e.g., Cartwright \& Collett, 1983). Previous research has shown that preschool children do not use the geometric relationships between objects in an array to reorient (Gouteux \& Spelke, 2001); however, it is possible that the objects used in those experiments were small and perceived as movable and unreliable. In one experiment, therefore, we investigated whether children reorient by a rectangular arrangement of tall and sturdy columns.

The second potential explanation appeals to the affordances of extended surfaces as barriers to vision or to locomotion (Kosslyn, Pick \& Fariello, 1974; Newcombe \& Liben, 1982). By small movements of the head or body, one can see or walk around a column or over an object, but one cannot see through or easily walk around a solid wall. ${ }^{1}$ In two experiments, we varied the height of the walls of a rectangular arena to investigate whether children's geometric encoding is only induced by walls that are high enough to obstruct vision or locomotion, or whether geometric encoding is induced equally effectively by walls that are low enough for children to see over and to traverse.

The third potential explanation appeals to the connectedness of the extended surface layout. The surface layout forms a unitary, connected whole, whereas columns, detached corners, or objects are separate entities whose geometric configuration must be constructed (Benhamou \& Poucet, 1998). It is possible, therefore, that children reorient by the shape of the surface layout because they can extract the geometric relationship from its unitary, connected form. One experiment (Gouteux \& Spelke, 2001) appears to cast doubt on this explanation, because children failed to reorient by the shape of a set of lines on the floor that were connected to form a unitary scalene triangle. Nevertheless, the lines in that experiment were presented along with a set of landmark objects that may have drawn children's attention away from the triangular form. Because children searched only at the landmark objects and did so no differently when the lines were absent than when they were present, it is not clear 
whether children even detected the lines in this experiment. In a final experiment, therefore, we tested children's reorientation in a room devoid of any competing cues with a unitary, rectangular pattern of connected two-dimensional lines surrounding the child on the floor.

The above three explanations have one feature in common: all appeal to processes that plausibly could have been shaped through years of experience in the indoor, closed environments in which modern children and laboratory animals live. Modern indoor environments typically are bounded by large, stable, and connected walls that serve as barriers to direct vision and locomotion. Modern outdoor environments also tend to be landscaped, with buildings and fences surrounding parks and streets. Children may become sensitive to these functional properties as they learn to navigate in such environments.

Alternatively, it is possible that processes of reorientation are built from mechanisms that develop independently of experience in these environments. Recent studies of animals, using controlled rearing methods, provide support for this suggestion (Brown, Spetch \& Hurd, 2007; Chiandetti \& Vallortigara, in press; Gray et al., 2005). In these studies, chicks and fish were raised, from the moment of hatching, either in a geometrically informative, rectangular environment or in a geometrically uninformative, circular environment. Then the two groups of animals were introduced into a rectangular environment, and their disoriented search behavior was observed. Although experience modulated animals' relative weighting of geometric and non-geometric information (Brown et al., 2007), animals in both rearing conditions showed high and equal sensitivity to the room-shape geometry.

These findings raise the possibility that human children's reliance on geometric information also depends, in part, on mechanisms attuned to the functional properties of the natural outdoor environments in which vertebrate navigation systems arose, rather than to the functional properties of the modern environments in which human children and laboratory animals live. In natural environments, connected, closed arrangements of walls that block vision and locomotion are rare: instead, the surrounding surface layout is an open and extended array of plains, hills, valleys or mountains. These contrasts motivated our tests for the environmental features guiding children's reorientation. 


\section{Experiment 1}

The first experiment investigated whether children reorient by the geometry of a rectangular enclosure with an open roof but walls that are high enough to prevent both locomotion and vision beyond the enclosure. Because such an environment is likely to be perceived as stable, as a barrier to vision and locomotion, and as a unitary, extended surface layout, all three functional explanations predict that children will use the shape of this enclosure to reorient themselves. Experiment 1 therefore provides a baseline against which each of the explanations can be tested.

Method

Subjects: Participants were 8 boys and 8 girls, ranging from 46 to 56 months of age. One additional child refused to enter the testing room without a parent and could not be tested.

Materials: The experiment was conducted in a circular chamber, $3.8 \mathrm{~m}$ in diameter, made of twelve white curved panels. One of these panels served as a door, which opened via an internal spring. The floor was solid gray, and the ceiling was equipped with symmetrically mounted lights and a hidden camera at the center. The room was protected from outside noise by soundproof walls and a second door. In the center of the chamber, 90-cm-high panels were connected to form a rectangular enclosure of dimensions $1.8 \mathrm{~m}$ by $1.2 \mathrm{~m}$ (see Figure 1); one panel swung open and served as a door allowing entry into the enclosure. Four small circular containers (diameter $10 \mathrm{~cm}$, height $3 \mathrm{~cm}$ ) were placed at the corners of the enclosure to serve as the hiding places for the search task. The whole experimental setup was symmetrical with respect to both the long and short axes of the rectangular structure.

Design and procedures: Each child was tested by an experimenter, while his or her parents watched and listened to the experiment from a video monitor just outside the testing room. First, the child was brought into the larger circular room, then into the smaller rectangular room where the rules of the game were explained. The child was given four search trials with a single hiding place. At the start of each trial, the experimenter hid a sticker under a container, and then pointed out the location of the door of the rectangular 
enclosure. Then a blindfold was placed over the child's eyes, and the child spun around in place several times until disorientation was confirmed by the child's inability to correctly point in the direction of the door. Next, the child was brought to the center of the room and turned to face one of the walls of the rectangular enclosure: a different wall on each of the four trials. Finally, the experimenter stood behind the child, removed the blindfold, and encouraged the child to search for the sticker. The location of the sticker and the order of the four facing directions were counterbalanced across children.

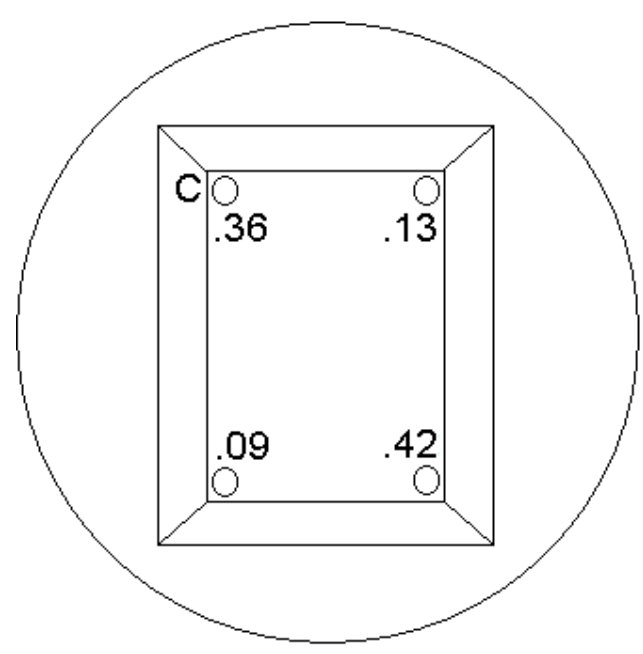

Experiment 1 $90-\mathrm{cm}$ walls

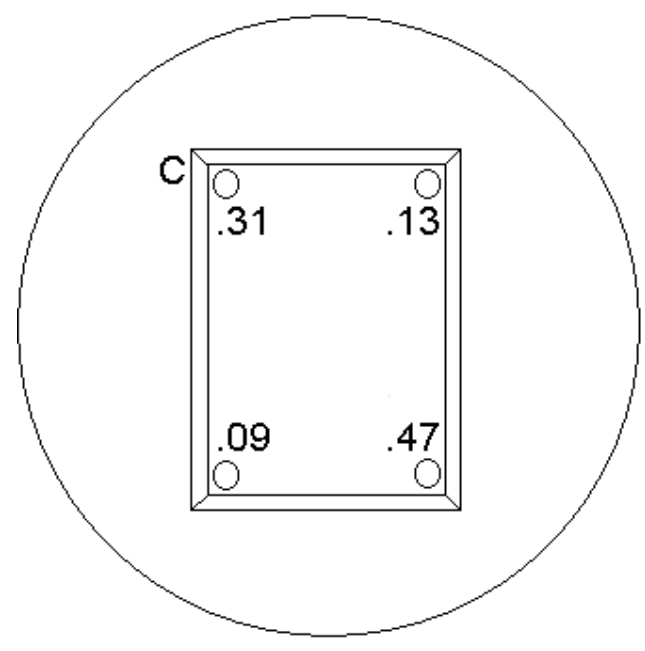

Experiment 3 $30-\mathrm{cm}$ walls

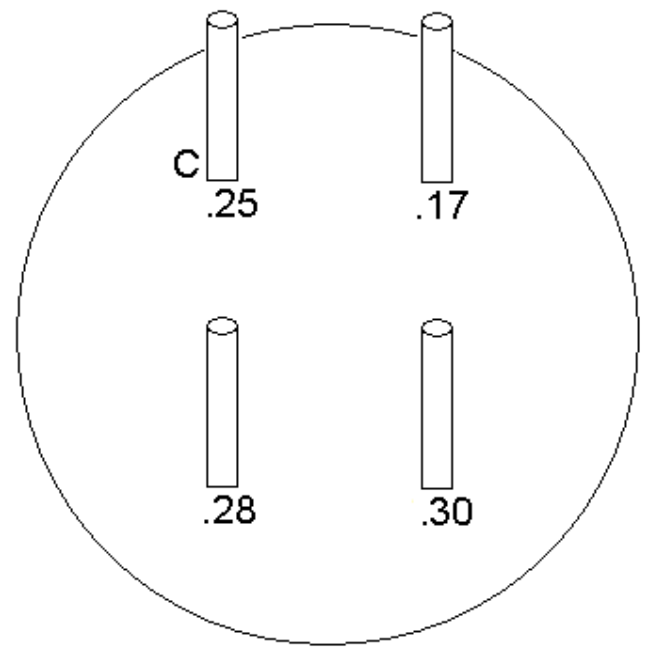

Experiment 2 Columns

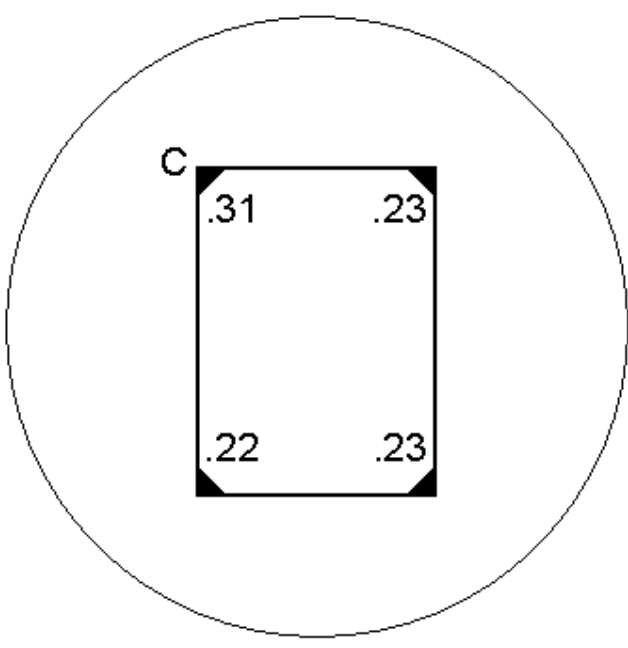

Experiment 4 Lines

$\underline{\text { ults }}$

Search rates at each corner are depicted in Figure 1. Because children searched 
equally at the correct and geometrically equivalent opposite corners, and they also searched equally at the geometrically incorrect near and far corners, we compared searches at the correct and opposite corners to searches at the near and far corners (see Figure 2). Children searched primarily at the two geometrically correct corners $(78 \%$, chance $=50 \%, \mathrm{t}(15)=6.260, \mathrm{p}<.001)$. No sex differences in performance were found.

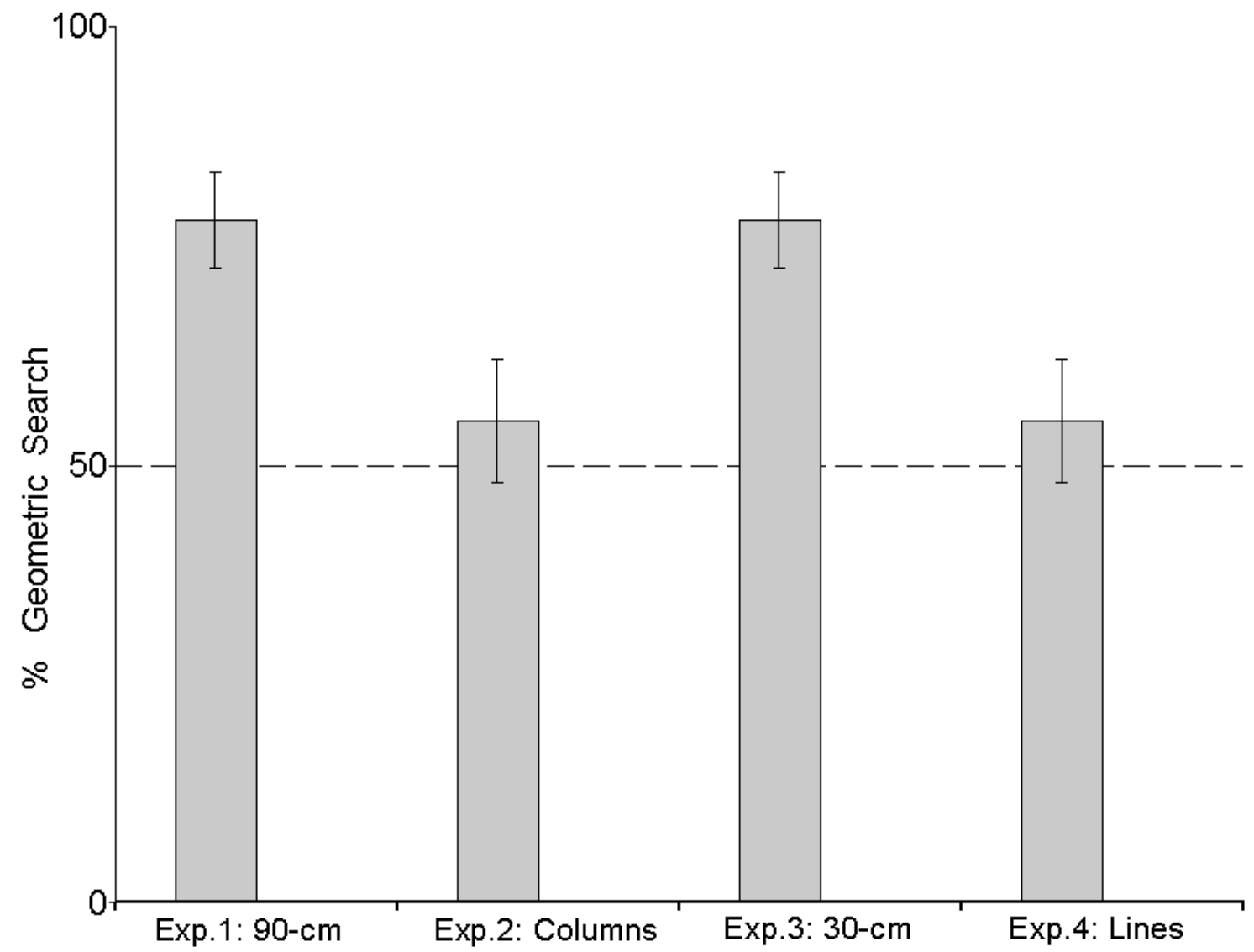

Discussion

Experiment 1 provided evidence that disoriented preschool children searched using the geometric information provided by a roofless enclosed arrangement of walls that were stable, connected, and served as barriers to vision and locomotion. The remaining experiments tested each of the three explanations for this effect.

\section{Experiment 2}

Experiment 2 investigated whether young children reorient by the geometry of an array of large landmarks that appear to be stable. The experiment was inspired by findings that honeybees learn the location of a food source in relation to the positions of large, free-standing 
columns (Cartwright \& Collett, 1983). Large columns such as trees and telephone poles are stable in human habitats as well. Using similar disorientation procedures as in Experiment 1, we tested children's reorientation within a rectangular arrangement of four large columns.

\section{Method}

The method was the same as in Experiment 1, except as follows. Participants were 8 boys and 8 girls, between 48 and 54 months of age. Four freestanding columns were arranged in a $1.2 \mathrm{~m}$ by $1.8 \mathrm{~m}$ rectangular array in the center of the circular room (see Figure 1 ). The columns were bright blue in color, $12 \mathrm{~cm}$ in diameter, and $1.8 \mathrm{~m}$ in height. Hiding places were small covered wells protruding from the side of each column, $15 \mathrm{~cm}$ above the floor. At the beginning of the study, the experimenter brought the subject into the circular room and then to the middle of the rectangular array. Children were instructed to try to stay in the center of the room; one child who strayed outside the area defined by the four columns was guided back into that space. The experimenter pointed out the door of the circular room before hiding the sticker in each trial, and disorientation was confirmed by the blindfolded child's inability to correctly point in the direction of the door after turning.

$\underline{\text { Results }}$

Search rates at each column are depicted in Figure 1. All children confined their search to the columns. Children searched equally at the four columns, however, showing no use of the informative geometry (55\% geometrically correct search, $\mathrm{t}(15)<1$; Figure 2$)$. Comparisons across experiments reveal a significant difference in geometric search performance between the 90-cm walls (Experiment 1) and the columns (Experiment 2), $\mathrm{t}(30)=2.736, \mathrm{p}=.01$. No sex differences were found.

\section{Discussion}

Disoriented children confined their search to the hiding wells of the four columns in the chamber, providing evidence that they detected and remembered the columns. Nevertheless, children failed to use the distance and sense relations between the columns to reorient and guide their search. These results provide evidence against the thesis that landmark size or stability is sufficient for children's use of geometric information for 
reorientation. Although disoriented children are adept at using large, stable objects as markers of a hidden object's location (Learmonth, Newcombe, Sheridan \& Jones, in press; see also Lee, Shusterman \& Spelke, 2006), they fail to use such objects as cues to their own geocentric orientation.

Why do children reorient by large walls but not by large columns? Whereas columns extend vertically, walls extend both vertically and horizontally. As a consequence, walls present a greater impediment to the vision and locomotion of land-dwelling animals such as humans. Moreover, columns are separate from one another, but the walls and surfaces in most indoor environments, including those used in most navigation experiments, are connected. Walls therefore define the boundary of the visible and navigable space itself. The next experiment was designed to test whether walls guide children's navigation because they serve as barriers to vision and locomotion.

\section{Experiment 3}

Experiment 3 tested whether children would reorient by the geometry of surfaces that were not barriers to vision or locomotion. Children were tested in a rectangular enclosure defined by a connected arrangement of surfaces, as in Experiment 1. In contrast to the high walls used in Experiment 1, however, the surfaces were just $30 \mathrm{~cm}$ high and therefore did not block children's view of the larger space or prevent them from walking throughout that space. To call children's attention to the latter feature, children entered the arena by stepping over it. If children reorient by the geometry of an arrangement of walls because that arrangement serves as a barrier to vision and locomotion, then the children in Experiment 3 should show less consistent use of geometry than those in Experiment 1.

\section{Method}

Participants were 8 boys and 8 girls between 46 and 51 months old. The method was identical to Experiment 2 and the enclosure was identical to Experiment 1, except that it contained no door and the height of its walls was reduced to $30 \mathrm{~cm}$. Children stepped over a wall to enter the enclosure, sometimes with a helping hand from the experimenter. 
$\underline{\text { Results }}$

Search rates at each corner are depicted in Figure 1. Children searched primarily at the two geometrically correct corners of the enclosure $(78 \%$ geometrically correct search, $t(15)=4.700, p<.001$, Figure 2). The rate of geometrically guided search was as high in this experiment as in Experiment $1(90 \mathrm{~cm}$ walls, $\mathrm{t}=0)$, and higher than in Experiment 2 (columns, $\mathrm{t}(30)=2.484, \mathrm{p}=.019)$.

\section{Discussion}

Children's searches were guided by the geometry of a layout of "walls" that were only $30 \mathrm{~cm}$ high. Because children were able to step and see over these enclosing surfaces, this finding suggests that the surface layout need not serve either as an obstacle to vision or as an barrier to locomotion in order for children to encode and use its geometry for reorientation. Like the rectangular enclosures in most previous experiments, however, the surfaces in Experiment 3 were connected to one another and formed a continuous geometric figure. Our final experiment investigated whether children's use of layout geometry depends on the unity and connectedness of the environmental features to which a geometric analysis applies. If so, three-dimensional surfaces may not be required for geometric reorientation.

\section{Experiment 4}

In Experiment 4, children were disoriented in the circular room while standing inside a two dimensional rectangular figure taped to the floor. To increase the salience of this figure, no hiding containers or other objects were placed in the room; covered, flat pockets at the corners of the figure served as the hiding places. If children's use of geometry depends on the unity of the layout feature to which a geometric analysis applies, then children should reorient by the shape of this two-dimensional form. If children detect the figure but fail to reorient by it, then they should confine their search to its four corners but not favor the two geometrically correct corners over the others. If children fail to detect the arrangement altogether, then they should either fail to search or choose locations unrelated to the lines and corners. 
Participants were 8 boys and 8 girls, between 47 and 53 months old. The method of Experiment 4 was identical to that of Experiments 2 and 3 except for the rectangular structure. The enclosure in Experiment 3 was replaced by black tape $(2 \mathrm{~cm}$ wide) on the floor, in the shape of a rectangle with the same dimensions as in the previous experiments. Also, instead of using the four containers as the hiding places (as in Experiment 1), the corners of the rectangular tape-figure were covered to form pockets that served as the hiding places (see Figure 1). Children were instructed to try to stay inside the rectangular space; one child who stepped outside the space was led back inside.

$\underline{\text { Results }}$

All children searched at the corners of the rectangular pattern on every trial. Nevertheless, children searched geometric and non-geometric corners equally often $(55 \%$ geometrically correct search, $\mathrm{t}(7)<1$, Figures $1 \& 2$ ). There were no sex differences. Comparing across experiments, children searched geometrically appropriate corners less consistently in Experiment 4 than in Experiment $1(90 \mathrm{~cm}$ walls $), \mathrm{t}(30)=2.736, \mathrm{p}=.01$, or Experiment $3(30 \mathrm{~cm}$ walls $), \mathrm{t}(30)=2.484, \mathrm{p}=.019$, and no differently than in Experiment 2 (columns), $\mathrm{t}<1$.

\section{Discussion}

Children confined their search to the corners of the rectangular arrangement of lines, providing evidence that they detected and remembered the lines and their intersection. Nevertheless, children did not reorient by the geometry of the overall two-dimensional pattern that these lines formed. These results are striking, because the lines on the floor indicated clearly the difference in the lengths and sense relations within the rectangular figure, and those relations were the same in this experiment as in its predecessors. Disoriented children evidently processed the two-dimensional figure differently from the three-dimensional surfaces in Experiments 1 and 3. The connectedness or salience of a rectangular arrangement is not sufficient for reorientation, when the arrangement is given by a two-dimensional pattern. 


\section{General Discussion}

The curious navigation patterns of disoriented children and laboratory animals has puzzled investigators for two decades: Why do disoriented rats focus on the subtle geometric properties of wall length and sense relations while often ignoring more salient properties such as wall color (black vs. white)? Why do disoriented children focus on the geometry of featureless extended surfaces but not that of interesting, salient objects? Many discussions of these navigational patterns appeal to the functional affordances of the environments in which children and laboratory animals live. Animals may rely on wall length, but not wall color, because walls define the space available for navigation (e.g., O'Keefe \& Burgess, 1996); children may reorient by the form of an arrangement of walls, but not of an arrangement of objects, because only the former are stable, unmovable landmarks (Learmonth, Newcombe \& Huttenlocher, 2001).

The present findings cast doubt on these accounts. The finding that $30-\mathrm{cm}-\mathrm{high}$ walls are sufficient for geometric encoding provides evidence that features of the layout need not serve as barriers to vision or locomotion in order to be used for reorientation. Moreover, children's failure to use the information in an array of large columns and in a salient, connected two-dimensional rectangular figure on the floor suggest that landmark stability or connectedness are not sufficient for children's reorientation. Instead, children reorient by the geometry of extended three-dimensional surface layouts.

Why do reorientation processes privilege three-dimensional surface layout geometry? One possibility is that the human navigation system is shaped not by the functional properties of the current, constructed environments in which children and laboratory animals live, but by the functional properties of the outdoor environments in which the navigation mechanisms of vertebrates emerged (see Gallistel, 1990). To reorient oneself effectively, a disoriented animal must rely on environmental properties that are unique, stable, and enduring. In natural, outdoor environments, surface colors and markings are not enduring, because of seasonal changes in vegetation and land covering. Large and fixed objects such as trees are stable and enduring, but their unique features usually are not sufficiently distinguishable and memorable 
to specify, to a lost and disoriented animal, which tree marks each specific location and direction (Cartwright \& Collett, 1983). Movable objects in nature may be even more difficult to discriminate and may change their location over time.

In contrast, the three-dimensional landscape of a natural environment—its hills and valleys, cliffs and streams - is stable, distinctive, enduring, and informative about all locations and directions in the environment. Barring major geological events, which occur rarely during the lifetime of any individual animal, surface layout geometry will be constant, when an animal visits the same environment at different times. Surface layout geometry is constant in modern environments as well, but it is a far less informative cue to reorientation, because the symmetrical shapes of most rooms render geometric information ambiguous. Thus, children appear to reorient by properties of the surface layout that are reliable and informative in the natural, outdoor environments in which their distant ancestors lived. On this interpretation, the present findings with children converge with the recent findings that birds and fish use geometry to reorient themselves, even if they are raised in an environment with no informative geometry whatever (Brown et al., 2007; Chiandetti \& Vallortigara, in press).

Beyond their contribution to research on human navigation, the present experiments may serve as a case study for testing theories in human evolutionary psychology. The mechanisms that guide navigation in human adults and children show strong homologies with the mechanisms guiding navigation in other animals (Cheng \& Newcombe, 2005), and the natural, outdoor environments in which navigation mechanisms emerged differ markedly from the man-made environments in which modern humans, and laboratory animals, live. These features of navigation create two avenues for teasing apart the ontogenetic and phylogenetic roots of human capacities: through controlled rearing studies with non-human animals, as in the work of Brown, et al. (2007) and Chiandetti \& Vallortigara (in press), and through studies that contrast the functional properties of natural and modern environments, as in the present research. Although the present research does not close the debate over the nature and development of the mechanisms of human navigation, we hope that it suggests a way to study their evolutionary roots in modern contexts. 


\section{$\underline{\text { References }}$}

Benhamou, S. \& Poucet, B. (1998). Landmark use by navigating rats (Rattus norvegicus):

Contrasting Geometric and featural information. Journal of Comparative Psychology, 112, 317-322.

Biegler, R. \& Morris, R. (1996). Landmark stability: Studies exploring whether the perceived stability of the environment influences spatial representation. Journal of Experimental Biology, 199, 187-193.

Brown, A. A., Spetch, M. L., \& Hurd, P. L. (2007). Growing in Circles: Rearing Environment Alters Spatial Navigation in Fish, Psychological Science, 18, 569-573.

Cartwright, B. A. \& Collett, T. S. (1983). Landmark learning in bees. Journal of Comparative Physiology A: Neuroethology, Sensory, Neural, and Behavioral Physiology, 151, 521-543.

Cheng, K. (1986). A purely geometric module in the rats' spatial representation. Cognition, 23, 149-178.

Cheng, K. \& Newcombe, N. S. (2005). Is there a geometric module for spatial reorientation? Squaring theory and evidence, Psychonomic Bulletin and Review, 12, 1-23.

Chiandetti, C. \& Vallortigara, G. (in press). Is there an innate geometric module? Effects of experience with angular geometric cues on spatial re-orientation based on the shape of 
the environment. Animal Cognition.

Gallistel, C. R. (1990). The Organization of Learning. Cambridge, M.A.: MIT Press.

Gouteux, S. \& Spelke, E. S. (2001). Children's use of geometry and landmarks to reorient in an open space. Cognition, 81, 119-148.

Gouteux, S., Thinus-Blanc, C., \& Vauclair, J. (2001). Rhesus monkeys use geometric and nongeometric information during a reorientation task. Journal of Experimental Psychology: General, 130, 505-519.

Gray, E. R., Bloomfield, L. L., Ferrey, A., Spetch, M. L., \& Sturdy, C. B. (2005). Spatial encoding in mountain chickadees: Features overshadow geometry. Biology Letters, 1, 314-317.

Hermer, L. \& Spelke, E. (1994). A geometric process for spatial reorientation in young children. Nature, 370, 57-59.

Hermer, L. \& Spelke, E. (1996). Modularity and development: The case of spatial reorientation. Cognition, 61, 195-232.

Kelly, D. M., Spetch, M. L., \& Heth, C. D. (1998). Pigeons' (Columba livia) encoding of geometric and featural properties of a spatial environment. Journal of Comparative Psychology, 112, 259-269.

Knierim, J. J., Kudrimoti, H. S., \& McNaughton, B. L. (1995). Place cells, head direction cells, and the learning of landmark stability. Journal of Neuroscience, 15, 1648-1659. 
Kosslyn, S. M., Pick, H. L., \& Fariello, G. R. (1974). Cognitive maps in children and men. Child Development, 45, 707-716.

Learmonth, A. E., Newcombe, N. S., Sheridan, N., \& Jones, M. (in press). Why Size Counts: Children's spatial reorientation in large and small enclosures. Developmental Science.

Learmonth, A. E., Newcombe, N. S., \& Huttenlocher, J. (2001). Toddlers' use of metric information and landmarks to reorient. Journal of Experimental Child Psychology, 80, 225244.

Lee, S. A., Shusterman, A., \& Spelke, E. S. (2006). Reorientation and landmark-guided search by young children: Evidence for two systems. Psychological Science, 17, 577-582.

Lew, A. R., Foster, K. A., \& Bremner, J. G. (2006). Disorientation inhibits landmark use in 1218-month-old infants. Infant Behavior \& Development, 29, 334-341.

Lourenco, S. F. \& Huttenlocher, J. (April, 2007). Coding location in enclosed spaces: Is geometry the principle? Paper presented at the biennial meeting of the Society for Research in Child Development, Boston, MA.

Newcombe, N. \& Liben, L. S. (1982). Barrier effects in the cognitive maps of children and adults. Journal of Experimental Child Psychology, 34, 46-58.

Newcombe, N. S. \& Ratliff, K. R. (2007) Explaining the development of spatial reorientation: Modularity-plus-language versus the emergence of adaptive combination. In J. Plumert \& J. Spencer (Eds.), The emerging spatial mind. Oxford University Press.

O'Keefe, J. \& Burgess, N. (1996) Geometric determinants of the place fields of hippocampal 
neurons. Nature, 381, 425-428.

Sovrano, V., Bisazza, A., \& Vallortigara, G. (2003). Modularity as a fish (Xenotoca eiseni) views it: Conjoining geometric and nongeometric information for spatial reorientation. Journal of Experimental Psychology: Animal Behavior Processes, 29, 199-210.

Vallortigara, G., Zanforlin, M., \& Pasti, G. (1990). Geometric modules in animals’ spatial representation: A test with chicks. Journal of Comparative Psychology, 104, 248-254. 


\section{Footnotes}

1 We thank Ulric Neisser for suggesting these possible explanations to us. 


\section{$\underline{\text { Figure Captions }}$}

Figure 1. Rates of search at each hiding place, with respect to the correct location (C).

Because the correct location was varied across subjects in the experiment, all data have been rotated prior to averaging and are displayed in this rotated form.

Figure 2. Percent of total search trials that are directed either to the correct location or to the geometric equivalent diagonal location. Chance rate is $50 \%$. 


\section{Acknowledgements}

We thank the members of the Laboratory for Developmental Studies at Harvard University for their suggestions and assistance in running these experiments. This research was supported by NIH grant HD-23103 to ESS. 